\title{
Frequency-domain full waveform inversion strategy using offset-weighted signals
}

\author{
Sérgio Luiz E. F. da Silva (Federal University of Rio Grande do Norte)*, Carlos A. N. da Costa (Federal University of Rio \\ Grande do Norte), Pedro Carvalho (Federal University of Rio Grande do Norte), João M. de Araújo (Federal University of Rio \\ Grande do Norte) and Gilberto Corso (Federal University of Rio Grande do Norte)
}

Copyright 2019, SBGf - Sociedade Brasileira de Geofísica.

This paper was prepared for presentation at the $16^{\text {th }}$ International Congress of the Brazilian Geophysical Society, held in Rio de Janeiro, Brazil, 19-22 August, 2019.

Contents of this paper were reviewed by the Technical Committee of the $16^{\text {th }}$ International Congress of the Brazilian Geophysical Society and do not necessarily International Congress of the Brazilian Geophysical Society and do not necessarily
represent any position of the SBGf, its officers or members. Electronic reproduction represent any position of the SBGf, its officers or members. Electronic reproduction
or storage of any part of this paper for commercial purposes without the written consent of the Brazilian Geophysical Society is prohibited.

\section{Abstract}

Full waveform inversion (FWI) is an imaging technique that brings high-resolution seismic images based on the inversion of the complete waveform. We investigate the frequency-domain finite difference FWI scheme of wide-aperture data as a tool to build good velocity models. Mathematically, the FWI is usually formulated as a least-squares optimization problem, i.e., minimize the square of the L2-norm between the modeled data and the recorded data of the seismic survey. The conventional least-square objective function suffers from the cycle-skipping effect that causes the solution to confined in a local minimum. To mitigate the cycle-skipping effect we balance the relative data contribution according to the source-receiver distance in which the weighting operation gives larger weight for the signals recorded in receivers close to the seismic source and lower weight for signals far from the seismic source that contains greater uncertainties in the estimation of traveltimes. Finally, we test the effectiveness of our inversion strategy using the acoustic Marmousi model. The proposed technique shows superior performance when compared to the conventional method.

\section{Introduction}

The oil industry is an important economic and technological activity of our society, Yilmaz (2001); King (1997). A big challenge in oil geophysics exploration is properly to image the subsurface, Virieux and Operto (2009). In recent years, the full wave inversion FWI method is largely being applied to the seismic image technique, Wang (2016). The context of the FWI is the seismic reflection exploration: a seismic source produces a wavelet-like wave that propagates and reflects in the geologic structures in the subsurface and, finally, is captured by receivers, Tarantola (1984). The main goal of the FWI method is to model the subsurface properties by computing traveling waveforms in the subsurface and comparing with the observed data at the receivers. The background of the FWI enterprise consists of the geophysical characteristics of the medium and the physical properties of the wave propagation, Fichtner (2010).

A mathematical complication of the FWI methodology is that the fitting technique behind the FWI is not well posed in the sense of Hadamar, see reference Hadamard (1902). The number of variables, typically the velocities of pixel elements in the medium, is not the same as the amount of variables recorded in the surface by the receivers. Thus, to mitigate this problem, we must include additional information, such as the solution softness by regularization methods. A standard strategy to compensate the disbalance between the number of model and recorded variables is to use many receivers and several shoots, Virieux and Operto (2009). However, the nonlinearity of the mathematics involved in the method and the intrinsic noise in the experimental setup pose additional complications to this technique, Fichtner (2010).

FWI is conventionally formulated as a least-squares fitting problem using local optimization techniques. This approach suffers from the cycle-skipping problem due to the presence of local minima and sensitivity to noise, specially when the starting model is not close to the true model, Virieux and Operto (2009). Several works have been proposed to mitigate the effects of the cycle skipping, Brossier et al. (2010). For example, Métivier et al. (2016) employed the Optimal transport (OT) distance to measure the misfit between modeled and observed data. The OT objective function shows very low sensitivity with respect to time-shifts of the seismic data. In the reference van Leeuwen and Herrmann (2013) it is proposed a novel and elegant formulation of FWI based on an iterative penalty method to progressively restrict an extended nonphysical model space to the physical model. Not all techniques use full seismogram information simultaneously. The naive idea that more information brings better resolution has also been challenged by two recent papers. The first work, de Freitas Silva et al. (2019), inspired in the sensory system of fishes, explored a selection of frequency bands in the FWI method. The second reference (Wang (2016), pp. 193-197), deals with a smart selection of receivers for an efficient FWI processing.

In a comparable research line, the work of Operto et al. (2006) propose the concept of weighting operator (W). The $\mathbf{W}$ is introduced to diminish in an efficient way the information of select receivers during the FWI processing. The $\mathbf{W}$ mitigate the contribution of the high-amplitude direct water wave, or long offset ocean-bottom data, during the inversion. The effect of small offsets can also be deleterious because they carry little information about the deep structures and may produce cycle skipping artifacts.

In this paper, we work with the weighting operator to improve seismic image resolution in the FWI context using the starting model with little information of the true model. This work challenges the idea that more 
information is always good to improve image resolution. The weighting operator selectively diminish information from unimportant receivers. The rest of this paper is organized as follows. In the Methodology we present in some detail the mathematics of the FWI and the design of the weighting operator. The numerical section is devoted to testing the weighting operator methodology developed in this paper. Finally, we conclude the work inserting the manuscript in a broad context and pointing out future research lines.

\section{Methodology}

The FWI is often formulated as an iterative least squares constrained optimization problem Virieux and Operto (2009):

$$
\min _{\mathbf{m}, \mathbf{u}} \frac{1}{2} \sum_{s, r}\left\|\mathbf{S}_{s, r} \mathbf{u}_{s}-\mathbf{d}_{s, r}\right\|_{2}^{2} \quad \text { s.t. } \quad A(\mathbf{m}) \mathbf{u}_{s}=\mathbf{q}_{s}
$$

where $\mathbf{m}$ represents the parameter of interest, $\mathbf{u}_{s}$ is the acoustic pressure wavefield for each source $s, \mathbf{S}_{s, r}$ represents the sampling operator (onto the receiver $r$ of the shot $s), \mathbf{d}_{s, r}$ is the observed data and $\|.\|_{2}$ denotes the Euclidean norm or L2-norm. In frequency-space domain, the constraint in (1) is a system of linear equations where $\mathbf{A}(\mathbf{m})$ is the discretized Helmholtz operator (impedance matrix) and $\mathbf{q}_{s}$ is the source term.

We define the residual data $\Delta \mathbf{d}_{s, r}=\mathbf{S}_{s, r} \mathbf{u}_{s}-\mathbf{d}_{s, r}$ by the difference between the modeled data and the observed data for each source-receiver pair. The constraint in equation (1) can be eliminated by assuming that the wave equation is satisfied at each iteration in optimization process, i.e., $\mathbf{u}_{s}=A^{-1}(\mathbf{m}) \mathbf{q}_{s}$. Thus, the least squares objective function can be compactly written by:

$$
\phi(\mathbf{m})=\frac{1}{2} \sum_{s, r} \Delta \mathbf{d}_{s, r}^{\dagger} \Delta \mathbf{d}_{s, r}
$$

We emphasize that $\Delta \mathbf{d}$ depends on the model parameters $(\mathbf{m})$. The symbol $\uparrow$ indicates the transpose conjugate (adjoint).

In this work, we propose the solution of this optimization problem using the weighted least-squares method, where the weights are related to the source-receiver distance. Thus, equation (2) is rewritten as follows:

$$
\phi(\mathbf{m})=\frac{1}{2} \sum_{s, r} \Delta \mathbf{d}^{\dagger} \mathbf{W} \Delta \mathbf{d}
$$

where $\mathbf{W}$ is a weighting operator applied to the data of the receivers. The idea behind $\mathbf{W}$ is to balance the relative contribution of observed data according to the source-receiver distance/offset $\left(\Delta O_{s r}\right)$. In this work, the weighting operator gives larger weight for the signals recorded in receivers near the seismic source and lower weight for signals far from the seismic source in each seismic experiment.

Amplitude loss with offset can be applied to each seismic trace within the operator $\mathbf{W}$ :

$$
W\left(\Delta O_{s r}\right)=\frac{1}{\left|\Delta O_{s r}\right|^{g}}
$$

where $\mathrm{g}$ is a scalar that controls the amplitude loss with respect to the source-receiver offset.

The derivative of $\phi(\mathbf{m})$ with respect to the model parameter m gives

$$
\frac{\partial \phi(\mathbf{m})}{\partial \mathbf{m}}=\nabla \phi(\mathbf{m})=\mathbb{R}\left\{\sum_{s, r}\left(\frac{\partial \Delta \mathbf{d}}{\partial \mathbf{m}}\right)^{\dagger} \mathbf{W} \Delta \mathbf{d}\right\}
$$

As we assume that the constraint in (1) is satisfied for all iterations, we can calculate the gradient of the objective function shown in equation (5) by adjoint-state method (Plessix (2006)). In this way, the gradient of the objective function is given by:

$$
\nabla \phi(\mathbf{m})=\mathbb{R}\left\{\sum_{s}\left\langle\lambda_{s}, \frac{\partial A(\mathbf{m})}{\partial \mathbf{m}} \mathbf{u}_{s}\right\rangle_{\mathbf{u}}\right\}
$$

where $\langle.\rangle_{\mathbf{u}}$ is the scalar product on all the wavefield elements $(\mathbf{u})$. In addition, $\lambda$ is the adjoint-state variable that is calculated by solving the adjoint wave equation given by:

$$
A^{\dagger}(\mathbf{m}) \lambda_{s}=-\sum_{r} \mathbf{S}_{s, r}^{\dagger}\left[\mathbf{W}\left(\mathbf{S}_{s, r} \mathbf{u}_{s}-\mathbf{d}_{s, r}\right)\right]
$$

Equation (7) corresponds to one adjoint system per shot. Note that $\lambda_{s}$ is the backpropagation of the term within the brackets in equation (7). In the classical FWI method, only the residual data is backpropagated. Here, the residual is weighted by $\mathbf{W}$.

\section{Numerical example}

To test the effectiveness of the inversion strategy proposed, we simulated a marine recording environment using the Marmousi model (Martin et al. (2006)) shown in Figure 1(a) where the water layer, on top of the model, is assumed to be known and kept constant at $1.5 \mathrm{~km} / \mathrm{s}$. The initial model is a smoothed version of the Marmousi model using a Gaussian smoother shown in Figure 1(b). The velocity models are $17.0 \mathrm{~km} \times 3.5 \mathrm{~km}$.

The acquisition geometry consists of a line of 46 equally spaced sources located every $375 \mathrm{~m}$ from lateral location equal (distance) to $15 \mathrm{~m}$ to $16,890 \mathrm{~m}$. For each source, it was employed 378 receivers located every $45 \mathrm{~m}$ from lateral location equal (distance) to $0 \mathrm{~m}$ to $16,965 \mathrm{~m}$ at the same depth of sources.

We generate data using a frequency-domain finite difference method. The Helmholtz operator was discretized on a grid with a spacing of $15 \mathrm{~m}$ using a classical 9point finite-differences with perfectly matched layer (PML) absorbing boundaries (P. Berenger (1994)). A Ricker wavelet centred on $5 \mathrm{~Hz}$ is used as the source term. The inversion process is performed by the multi-scale approach to mitigate the non-linearity of the inversion problem (Carey Bunks (1995)) using an I-BFGS method (Nocedal and Wright (2006)) to invert the data in three frequency bands: [3 4 4 5], [ [ $\left.\begin{array}{lll}6 & 7 & 8\end{array}\right]$ and [9 10 11] Hz. For each frequency band, we computed 20 l-BFGS iterations.

The maximum source-receiver offset was 16,950 meters. We used $g=1$ and with that the weights ranged from 0.06 
(a)

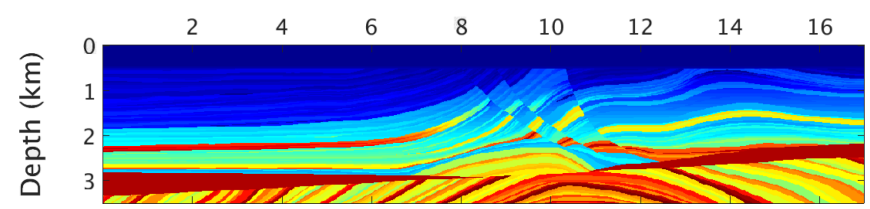

(b)

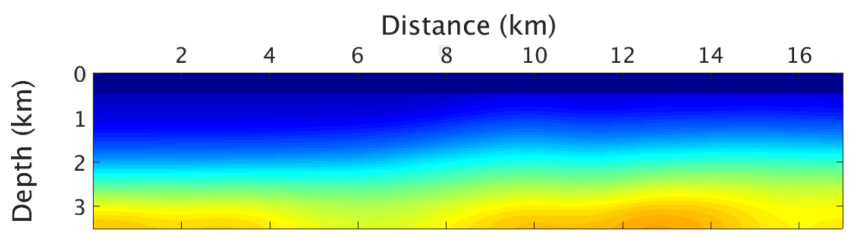

(c)

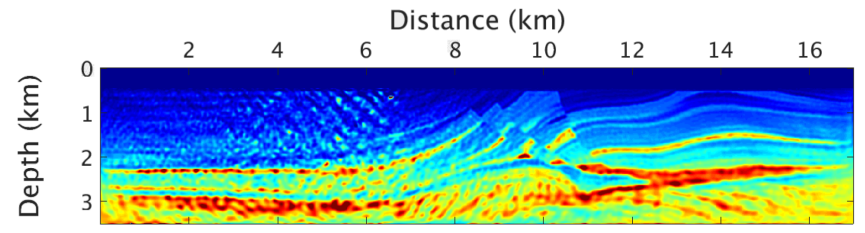

(d)

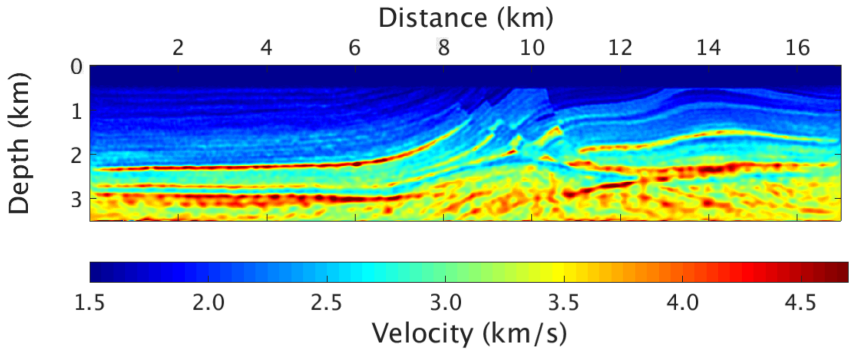

Figure 1: a) Marmousi velocity model (True model); b) Marmousi smooth velocity model (Initial model); Inversion results $\mathrm{c}$ ) standard approach and d) strategy proposed.

to 66.67. Thus, the weighting operator in the data space with the largest source-receiver offset has a value of 0.06 and a value of 66.67 for the smaller source-receiver offset, following equation (4).

The inversion result for the standard approach is shown in Figure 1(c). Note that, inversion with the conventional processing fails to provide a good reconstruction but the proposed method gives a result closer to the true model (Figure 1(d)). Conventional FWI generated many artifacts in the region with a distance (lateral location) of less than $8 \mathrm{~km}$ in all depths, mainly in the shallower structures of the model. This happens because in this region there are a series of hydrocarbon layers with velocity abrupt changes and gas charged sand channel into the structural model generating large uncertainties in the seismic signals recorded over long distances, since the initial model was not reasonably close to the true model. For example, consider a shot into this region and a receiver ten kilometers away. The irradiated wave is rescued after passing through a series of complex structures that the initial model was not aware of, such as the three faults in the central part of the Marmousi model, strengthening the cycle-skipping effect. In comparison, the estimated velocity model using the method proposed is significantly closer to the true model and there isn't artifacts.

\section{Conclusions}

In this paper, we explore the concept of balancing information in the solution of a ill-posed mathematical problem and compare with the FWI conventional. We present a strategy to diminishing the contribution of the receivers far from the source during the $\mathrm{FWI}$ inversion to mitigate the cycle-skipping effect. The numerical example demonstrate the advantages of the strategy proposed. In contrast to conventional method, the strategy proposed diminishes the influence of cycle-skipping artifacts, which suggests that the presented strategy may be a good choice when using wide-aperture seismic data because the arrival times was well estimated. Note that in the region between $2 \mathrm{~km}$ and $3 \mathrm{~km}$ depth and 12 and $16 \mathrm{~km}$ distance, the salt layer is identified using both methods, however, in the conventional FWI this region becomes more evident because the data amplitude information is related to reflectivity. This information was attenuated in the method proposed by the weighting operator. For this reason, a study about how to vary the value of $g$ during the inversion process should be done so that the deeper structures are better determined using the diving waves.

\section{ACKNOWLEDGMENTS}

The authors gratefully acknowledge support from Shell Brasil through the "New Methods for Full Waveform Inversion" project at the Universidade Federal do Rio Grande do Norte and the strategic importance of the support given by ANP through the R\&D levy regulation. The authors also gratefully acknowledge the support from Brazilian National Council for Scientific and Technological Development (CNPq).

\section{References}

Brossier, R., Operto, S., and Virieux, J., 2010, Which data residual norm for robust elastic frequency-domain full waveform inversion?Robust full waveform inversion: Geophysics, 75, no. 3.

Carey BunksFatimetou M. Saleck, S. Z. G. C., 1995, Multiscale seismic waveform inversion: Geophysics, 60, no. $5,1457-1473$.

de Freitas Silva, F. W., da Silva, S. L. E. F., Henriques, M. V. C., and Corso, G., 2019, Using fish lateral line sensing to improve seismic acquisition and processing: PLOS ONE.

Fichtner, A., 2010, Full Seismic Waveform Modelling and Inversion: Springer Verlag.

Hadamard, J., 1902, Sur les problèmes aux dérivés partielles et leur signification physique: Princeton University Bulletin, 13, 49-52.

King, P. R., 1997, Physics boosts oil production: Physics World, 10, 33-37.

Martin, G. S., Wiley, R., and Marfurt, K. J., 2006, Marmousi2: An elastic upgrade for marmousi: The Leading Edge, 25, no. 2, 156-166. 
Métivier, L., Virieux, J., Brossier, R., Mérigot, Q., and Oudet, E., 2016, Measuring the misfit between seismograms using an optimal transport distance: application to full waveform inversion: Geophysical Journal International, 205, no. 1, 345-377.

Nocedal, J., and Wright, S. J., 2006, Numerical optimization: Springer, New York, NY, USA, second edition.

Operto, S., Virieux, J., Dessa, J.-X., and Pascal, G., 2006, Crustal seismic imaging from multifold ocean bottom seismometer data by frequency domain full waveform tomography: application to the eastern-nankai trough.: Journal of Geophysical Research, 111, B09306.

P. Berenger, J., 1994, A perfect matched layer for the absorption of electromagnetic waves: Journal of Computational Physics, 114, 185-200.

Plessix, R.-E., 2006, A review of the adjoint-state method for computing the gradient of a functional with geophysical applications: Geophysical Journal International, 167, no. 2, 495-503.

Tarantola, A., 1984, Inversion of seismic reflection data in the acoustic approximation: Geophysics, 49, 1259 1266.

van Leeuwen, T., and Herrmann, F. J., 2013, Mitigating local minima in full-waveform inversion by expanding the search space: Geophysical Journal International, 195, no. 1, 661-667.

Virieux, J., and Operto, S., 2009, An overview of full-waveform inversion in exploration geophysics: Geophysics, 74, WCC1-WCC26.

Wang, Y., 2016, Seismic inversion: Theory and applications: Wiley Blackwell.

Yilmaz, O., 2001, Seismic data analysis: Processing, inversion, and interpretation of seismic data.: Society of exploration geophysicists. 